\title{
Autoantibody-dependent amplification of inflammation in SLE
}

\author{
Hantao Lou ${ }^{1,2}$, Beata Wojciak-Stothard ${ }^{3}$, Marieta M. Ruseva ${ }^{4}$, H. Terence Cook ${ }^{4}$, Peter Kelleher ${ }^{2,5}$, Matthew C. Pickering ${ }^{4}$, \\ Juthathip Mongkolsapaya ${ }^{1,6}$, Gavin R. Screaton ${ }^{1,6}$ and Xiao-Ning X ${ }^{2}$
}

\begin{abstract}
Anti-double stranded DNA antibodies (anti-dsDNA) are a hallmark of SLE but their role in disease pathogenesis is not fully resolved. Anti-dsDNA in serum are highly heterogeneous therefore in this study, we aimed to dissect the functional specificities of anti-dsDNA using a panel of human monoclonal antibodies (humAbs) generated from patients with active lupus nephritis. A total of 46 ANA reactive humAbs were isolated and divided into four broad classes based on their reactivity to histones, DNA and Crithidia. Functional analysis indicated that one subclass of antibodies bound strongly to decondensed DNA areas in neutrophil extracellular traps (NETs) and protected NETs from nuclease digestion, similar to the sera from active SLE patients. In addition, these anti-dsDNA antibodies could stimulate type I interferon responses in mononuclear phagocytic cells, or NF-kB activity in endothelial cells, by uptake of NETs-anti-NETs immune complexes and subsequently trigging inflammatory responses in an Fc-gamma receptor (Fcg-R)-dependant manner. Together our data suggest that only a subset of anti-dsDNA antibodies is capable to amplify inflammatory responses by deposit in the nephritic kidney in vivo, protecting NETs digestion as well as uptake of NETs immune complexes into Fcg-R-expressing cells in vitro.
\end{abstract}

\section{Introduction}

Systemic lupus erythematosus (SLE) is a multi-system autoimmune disease of varying severity, which commonly affects the skin and joints, but can also affect a variety of other organ systems. Most patients with SLE develop antinuclear antibodies (ANA) directed to a variety of nuclear components. Anti-dsDNA antibodies are highly specific, being found in $68-83 \%$ of SLE patients at some time during their illness ${ }^{1-3}$. Anti-dsDNA antibodies are associated with active SLE disease and deposit in the kidney of some lupus nephritis (LN) patients, which may contribute to the development of lupus nephritis $(\mathrm{LN})^{4-11}$. There are several proposed mechanisms whereby anti-dsDNA can

Correspondence: Hantao Lou (h.lou13@Imperial.ac.uk) or Xiao-Ning Xu (x.xu@imperial.ac.uk)

'Division of Immunology and Inflammation, Department of Medicine, Hammersmith Campus, Imperial College London, London W12 0NN, UK ${ }^{2}$ Department of Medicine, Centre for Immunology \& Vaccinology, Chelsea and Westminster Hospital, Imperial College London, London SW10 9NH, UK Full list of author information is available at the end of the article.

Edited by T. Kaufmann cause inflammation and contribute to disease pathogenesis, including the formation of immune complexes with chromatin exposed on apoptotic cells and the subsequent recruitment of Fcg-R myeloid cells or by the fixation of complement.

NETosis is a neutrophil-specific cell process (formation of NET occurs by two main pathways: a slow neutrophilspecific lytic cell death pathway and a more rapid nonlytic pathway from viable cells), which is characterized by the formation of NET in response to infectious (for example C. albicans) and sterile stimulation (for example anti-phospholipid antibodies, anti-RNP antibodies, IL-8 and monosodium uric acids) $)^{12-14}$. NETs are composed of decondensed chromatin and a range of neutrophil granular antimicrobial proteins including myeloperoxidase (MPO), neutrophil elastase (NE), LL37, etc. NETs have an important role during infection as they capture and neutralise pathogens ${ }^{13,14}$. Impairment in the clearance of apoptotic cells and NETosis are believed to be a potential source of autoantigens in SLE, which are proposed to 
trigger systemic autoimmune inflammation ${ }^{10-12}$. In this regard, it has been shown that activation of neutrophils by immune complexes containing nucleic acid can promote expression of pro-inflammatory cytokines, including type I interferon SLE ${ }^{13-15}$. Impairment of NETs degradation was observed in SLE patients and the undigested NETs was found to deposit in the kidney of some Lupus nephritis patients ${ }^{10,16-18}$. This suggests that impaired NETs digestion is associated with renal damage in SLE patients.

To gain more insight into the nature of the interaction between NETs and autoantibodies in lupus we set out to generate human monoclonal antibodies (humAb) from lupus patients that react with NETs. We generated a panel of 46 ANA reactive humAb and characterise their reactivity to dsDNA, histones and Crithidia luciliae antigen. A subset of five of these anti-ANA humAb was fully polyreactive, binding strongly to dsDNA, histone 1 and Crithidia. These five humAb also bind to NETs and inhibit NETs degradation. Furthermore, this subset of five humAb when in complex with NETs promote type I interferon expression upon uptake by blood monocytes and increases NF-kB expression in endothelial cells in an Fcg-Rdependent manner, providing a potential mechanism for autoantibody-driven amplification of inflammation.

\section{Materials and methods}

\section{Patients, blood samples and donor cells}

Blood samples were collected from two patients with active renal SLE for single plasmablast molecular cloning. Serum samples were also collected from patients undergoing diagnostic blood tests for suspected connective tissue disease and from SLE patients who were being monitored for active disease. The study was performed in accordance with the local Research Ethics Committee and Institutional approval. Sera from 65 patients with SLE were obtained from the Department of Infection and Immunity at North West London Pathology Trust Charing Cross Hospital. Ten ANA-ve patients were also recruited as negative controls. All SLE patients fulfilled the 1997 revised criteria of the American College of Rheumatology for the diagnosis of SLE. Clinical data on disease manifestations, treatments. renal function, ANA titre, complement and anti-dsDNA levels were collected from patient records. SLE disease activity was classified on the basis of symptoms, alteration in renal function and SLE treatment using the BILAG2004 criteria for constitutional, cutaneous, musculoskeletal and renal disease. Active disease was defined as the need to commence or increase cytotoxic, steroid and hydroxychloroquine therapy.

Peripheral blood mononuclear cells (PBMC) were isolated by Ficoll-Hypaque density-gradient centrifugation from healthy donors. Monocyte was isolated by CD14 magnetic beads while neutrophils were isolated by dextran sedimentation.

\section{Isolation of monoclonal antibodies from plasmablasts}

Monoclonal antibodies were isolated from single plasmablasts. Briefly, plasmablasts (CD3-CD20 $0^{\mathrm{low}} /$ $\mathrm{CD} 19^{+} \mathrm{CD} 38^{\text {hi }} \mathrm{CD} 27^{\text {hi }}$ ) were sorted by a FACS sorter (BD FACSARIA) into 96-well plates containing $10 \mu$ l RNaseinhibiting RT-PCR catch buffer ( $5 \mathrm{ml}$ RNase-free water, $50 \mu \mathrm{l} 1 \mathrm{M}$ Tris $\mathrm{pH} 8$ and $125 \mu \mathrm{l}$ RNasin (Promega)). Plates were immediately sealed and frozen on dry ice and stored at $-80^{\circ} \mathrm{C}$. Single-cell cDNA was synthesised in the original sort plates by adding $15 \mu \mathrm{l}$ RT-PCR reaction mix. RT-PCR reaction mix contains $1 \mu$ forward primer mix $(1.2 \mu \mathrm{M}), 1 \mu \mathrm{l}$ reverse primer mix $(1.2 \mu \mathrm{M}) 1 \mu \mathrm{l}$ dNTPs $(200 \mu \mathrm{M}), 5 \mu \mathrm{l} 10 \times$ buffer, $0.5 \mu \mathrm{l} \mathrm{PCR}$ enzyme mix and $6.5 \mu \mathrm{H}_{2} \mathrm{O}$. Individual $\operatorname{IgH}$ and $\operatorname{IgL}(\mathrm{k}$ or $\lambda)$ genes were amplified in the second round PCR reaction with HotstarTaq PCR kit. IgH and Ig $\lambda$ genes are digested with AgeI/SalI or AgeI/XhoI, respectively, prior to the ligation into the variable gene cloning site of the $\mathrm{IgH} / \mathrm{IgL}$ expression vectors. The expression vectors are composed of the appropriate human constant region downstream of a murine immunoglobulin signal peptide and ampicillinresistant gene. The Igk products were sequenced and amplified according to its gene family by another round of PCR. They were then digested with AgeI/BsiWI before ligated into Igk expression vector. IgH and IgL chaincontaining plasmids were mixed with PEI (Polyethylenimine) and transfected into human embryonic kidney fibroblast 293T cells. Cells were washed with DMEM/PBS $24 \mathrm{~h}$ after transfection and then cultured in protein-free media Supernatants are collected five-six days after transfection.

\section{Antibody purification}

293T cells transfected with VH and VL plasmids were cultured in PF Ultradoma (Lonza) for 5 days before supernatant was collected, filtered $(0.2 \mu \mathrm{m})$ and treated with $\mathrm{MNase}(2.5 \mathrm{U} / \mathrm{ml})$ for $120 \mathrm{~min}$. The supernatant is then loaded to a column packed with protein A beads and washed with PBS three times before eluted with glycine $\mathrm{pH} 2.7$ and neutralised with Tris- $\mathrm{HCl} \mathrm{pH}$ 8. The purified antibody is subsequently buffer-exchanged with PBS and stored in $-80^{\circ} \mathrm{C}$.

\section{Determination of ANA, DsDNA and anti-histone antibodies}

ANA-IIF screens on serum samples obtained from patients with suspected or know SLE was performed manually on Hep-2 cells (ANA-IIF) according to the manufacturer's protocol (IMMCO Diagnostics, Ely, UK) and a titre of $\geq 1: 160$ was considered positive, leading to further antigen characterisation. ANA-IIF screen were performed on neat supernatant cultures of monoclonal 
antibodies isolated from SLE patients. Anti-DsDNA antibodies were detected by ELISA (ED-FDNA 100) according to the manufacturer's instructions. Confirmation of positive DNA samples was performed using the Crithidia luciliae immunofluorescence (CLIF) test (BioSystems, Barcelona Spain). Anti-histone and antichromatin (ORGENTEC) ELISAs were performed according to the manufactures' manual. Anti-histone/ chromatin western blot protocol is described in the supplementary methods.

\section{Apoptotic cells binding}

Jurkat cells were cultured in RPMI $+10 \%$ fetal calf serum (R10) and washed to be resuspended in RPMI at a concentration of $1 \times 10^{6}$ cells $/ \mathrm{ml}$. Jurkat cells were then added to 6 -well plate at $1 \mathrm{ml} /$ well. For anti-Fas IgM killing, $100 \mathrm{ng} / \mathrm{ml}$ anti-Fas IgM was added to each well and the Jurkat cells were collected, washed after $60 \mathrm{~min}$. The treated Jurkat cells were rested in R10 for $8 \mathrm{~h}$. For UV killing, the plate was placed under UV (wavelength $320 \mathrm{~nm}$ ) for $30 \mathrm{~min}$ followed by washing. The cells were cultured in R10 for $3 \mathrm{~h}$. Apoptotic Jurkat cells were then co-incubated with the anti-dsDNA mAbs $(5 \mu \mathrm{g} / \mathrm{ml})$ for $1 \mathrm{~h}$ at $4{ }^{\circ} \mathrm{C}$ followed by FITC-conjugated anti-human IgG (Sigma), Annexin V and PE staining. The mAb binding to the apoptotic cells was analysed by flow cytometer and microscope.

Generation and analysis of NETs binding and degradation

Neutrophils from healthy donors were isolated by dextran sedimentation according to standard protocols. Sterile 13-mm-round-glass coverslips were placed on 24well plates and $5 \times 10^{5}$ neutrophils were seeded on each well for $30 \mathrm{~min}$ at $37^{\circ} \mathrm{C}$. $500 \mathrm{ng} / \mathrm{ml}$ PMA or A23187 solution in RPMI was added to the wells to stimulate the neutrophils for $3 \mathrm{~h}$. NETs were induced as described and co-incubated with either PBS or mAbs at various concentrations for $1 \mathrm{~h}$ and then washed with PBS. $1 \mathrm{U} / \mathrm{ml}$ Mnase or DNaseI was then added to the NET with $2 \mathrm{mM} \mathrm{CaCl}_{2}$ to kick start the digestion for $10 \mathrm{~min}$ at $37^{\circ} \mathrm{C}$. The supernatant was then transferred to a black plate and sytox orange added to the wells. The amount of digested DNA was then measured by fluorescence spectrometry. The number of NETs digested with $1 \mathrm{U} / \mathrm{ml}$ Mnase in PBS is defined as $100 \%$ digestion.

Neutrophil extracellular traps (NETs) were fixed with $4 \%$ paraformaldehyde and blocked with $5 \%$ goat serum. NET were then co-incubated with $10 \mu \mathrm{g} / \mathrm{ml} \mathrm{mAb}$ followed by FITC-conjugated anti-human IgG. To visualise NETs, DAPI was added to the slides. Images were taken by fluorescent microscopy (OLYMPUS IX70).

During NETosis, chromatin becomes decondensed and relaxed to form long stretches. DNA-intercalating dye stains condensed DNA strongly but reacts weakly with decondensed DNA ${ }^{19}$. Serum binding to long stretches of decondensed DNA (denoted by arrows on Fig. 1) was visualised by anti-human IgG FITC. The decondensed DNA binding ability of serum (1:100) was scored double blindly by two immunologists at a grade of 0,1 and $2^{20,21}$.

\section{Measurement of type I interferon secretion by monocytes}

NETs were induced using the described protocol, washed with PBS and co-incubated with mAbs at the indicated concentrations for $1 \mathrm{~h}$ at $37^{\circ} \mathrm{C}$. NETs were then co-incubated with human monocytes isolated from healthy donors in RPMI-10 per cent FBS (fetal bovine serum). After $24 \mathrm{~h}, \mathrm{mRNA}$ was isolated (RNeasy Plus Mini Kit, Qiagen) followed by cDNA synthesis (SuperScript IV, Thermofisher) and gene expression was measured by qRT-PCR (brilliant III SYBR Green, Agilent). Primers used IFNA1 (forward: 5'-GGAGTTTGATGGCAACCA GT-3', reverse: 5'-CTCTCCTCCTGCATCACACA-3'), IFNB (forward: 5'-AGCACTGGCTGGAATGAGAC-3', reverse: $5^{\prime}$-TCCTTGGCCTTCAGGTAATG-3').

\section{Endothelial cells NFKB luciferase reporter assay}

HPAECs passaged between 3-6 passages were seeded into 96-well culture plates at $2 \times 10^{4}$ cells/well. After reaching $90 \%$ confluence, HPAECs were infected with adenoviruses containing $\mathrm{NFK}_{\mathrm{K}} \mathrm{B}$ luciferase reporter construct (AdNFkB-luc) ${ }^{4}$ at multiplicity of infection (MOI) 1:100. $4 \mathrm{~h}$ post-infection, the medium was replaced with $50 \mu \mathrm{l}$ of (ECGM-2) media, containing $0.15 \mu \mathrm{g} / \mathrm{ml}$ of NETs DNA with, or without control, dsDNA and LALA antibodies $(100 \mu \mathrm{g} / \mathrm{ml})$ Cells were then incubated for a further $24 \mathrm{~h}$ at $37^{\circ} \mathrm{C}$. NETs were pre-incubated with antibodies for $30 \mathrm{~min}$ at room temperature before addition to cells. Treatment with $10 \mathrm{ng} / \mathrm{ml}$ of TNF- $\alpha$ (Sigma) was used as a positive control for NF- $\mathrm{kB}$ activation. After $24 \mathrm{~h}$ incubation, cells were lysed with $1 \times$ lysis buffer (Promega) and $20 \mu \mathrm{l}$ of each sample was transferred to white 96-well plates (Corning ${ }^{\mathrm{TM}}$ ) and combined with $100 \mu \mathrm{l}$ of Luciferase Assay Reagent ${ }^{\mathrm{TM}}$ (Promega,). Luminescence, proportional to the level of NFkB-driven expression of luciferase, was measured in the Glomax ${ }^{\mathrm{TM}}$ luminometer.

\section{Accelerated nephrotoxic nephritis (NTN) mice model}

C57BL/6 wild-type mice were purchased from Harlan Ltd. (Bicester, UK). All animal procedures were performed in accordance with institutional guidelines and under license by the UK government.

Accelerated NTN (ANTN) was induced by the i.v. injection of $200 \mu \mathrm{l}$ of sheep nephrotoxic serum (a sheep Ig fraction containing anti-mouse GBM antibodies) into mice that had been sensitised with an intraperitoneal injection of $200 \mu \mathrm{g}$ of sheep IgG (Sigma-Aldrich, Dorset, UK) in CFA (Sigma-Aldrich). Mice were housed in SPF conditions and individually ventilated cages. 
A

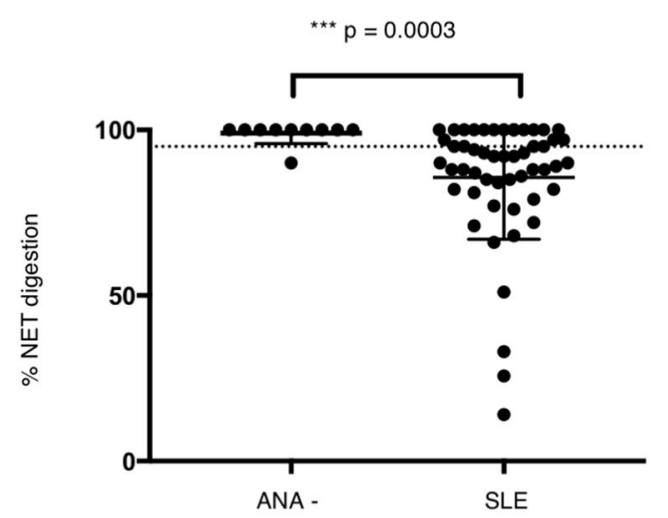

B Inactive SLE patient serum Anti-dsDNA titre: 170

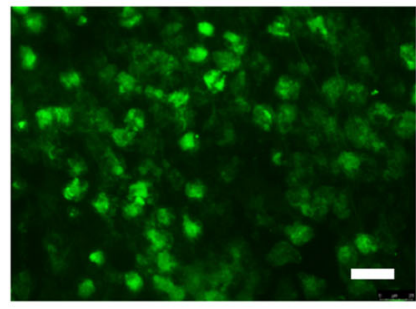

$100 \%$ NET digestion
Active SLE patient serum Anti-dsDNA titre: 125

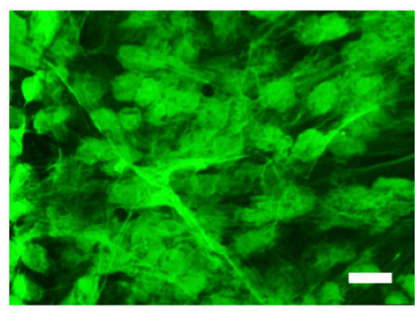

$49 \%$ NET digestion
C
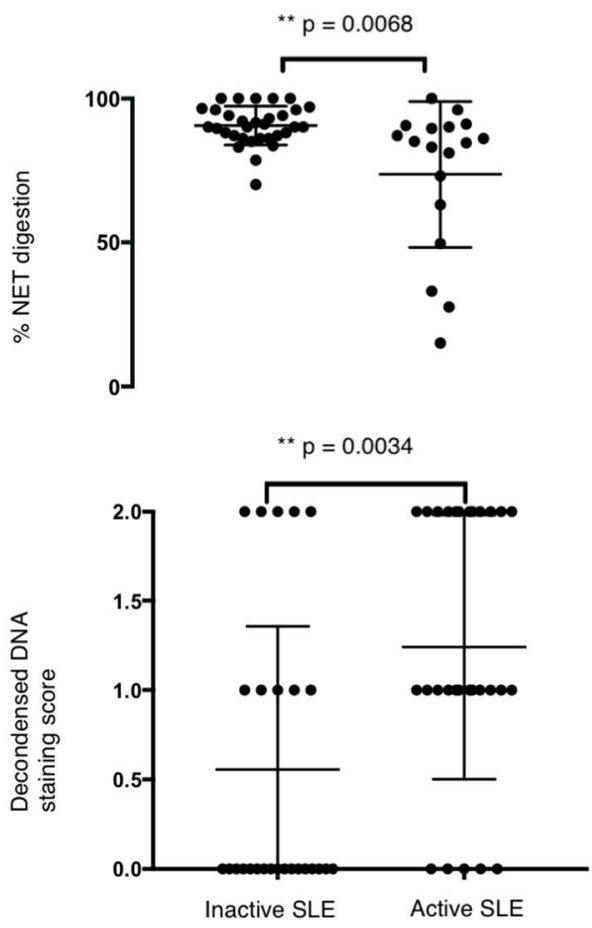

Fig. 1 Serum from some active SLE patients protect NET from digestion. a NET digestion ability of ANA -ve donors $(n=10)$ and SLE patients $(n$ $=51$ ) were measured and compared. NET were stimulated by PMA followed by incubation with $10 \%$ serum for $6 \mathrm{~h}$, and the amount of DNA in the supernatant was measured. $3 \mathrm{U} / \mathrm{ml}$ Mnase in PBS was used to digest NET, and the amount of digested NET is regarded as 100\%. The percentage of NET digestion was calculated accordingly. Data represent mean of three repeats \pm SEM, Mann-Whitney U. b NET binding of sera (10\%) from 51 patients were studied by immunofluorescence microscopy. Representative images of inactive and active SLE serum (10\% serum diluted in PBS) binding are illustrated $(n=51)$. The serum binding to NET was visualised by FITC-conjugated anti-human IgG antibodies. c NET digestion ability of active $(n=18)$ and inactive SLE patients $(n=33)$ was measured three times and compared. NET staining was scored for each SLE patients in a double-blind manner and analysed on a dot plot $(n=51)$. Data represent mean of three repeats, Mann-Whitney $U$.

\section{Kidney tissue staining}

Anti-dsDNA monoclonal antibodies $(200 \mu \mathrm{g} /$ mice $)$ were intraperitoneally into nephrotoxic nephritis (NTN) mice. After $6 \mathrm{~h}$, the mice were killed and serum and kidney was harvested. Kidney samples were fixed with OCT and $2 \mu \mathrm{m}$ sections were cut and stored at $-80^{\circ} \mathrm{C}$. For staining, slides were soaked in acetone for 7 min, dried, washed with PBS and incubated with 5\% goat serum (Sigma G9023-10 ml) for $1 \mathrm{~h}$. Slides were dried and stained with anti-human/mouse IgG FITC diluted at 1:200 in blocking buffer (goat anti-human Fc-specific FITC, Sigma 100M4848 F5387) for $1 \mathrm{~h}$ at room temperature. Finally, slides were washed three times with PBS and mounted with the mounting media for visualisation under a microscope.

\section{Statistical analysis}

All experiments were repeated at least in triplicate with four experimental repeats. Data are presented as means \pm standard error deviation of the mean (SEM). All graphs and calculations were carried out with GraphPad Prism6 version 6.03 for Windows (GraphPad Software Inc). Oneway ANOVA with Tukey multiple comparison post-test was used to determine statistical significance.

\section{Results}

Several laboratories have reported that serum from some SLE patients can impair NETs digestion, implying the presence of a nuclease inhibitor and/or anti-NETs antibodies, which can either prevent access of endogeneous nucleases to or inhibit their activity on NETs. We measured NET nuclease-protection activity in polyclonal sera (10\% neat serum is diluted in PBS) from a cohort of SLE patient (serum anti-DNA titre and NETs protection ability is provided in Supplementary Table 1). The patients were scored by BILAG into active and inactive SLE groups (as described in Methods). The ANA-ve control patients (confirmed by ANA staining and dsDNA ELISA test) were also enrolled as a negative control from Charing Cross Hospital. 


\section{Active SLE patients show stronger binding to decondensed DNA of NETs}

Fifty-one SLE patients sera were tested and ANA+ve SLE patient serum (10\% serum diluted in PBS) displayed significantly lower NETs digestion than control ANA-ve serum in the presence of DNase (Fig. 1a). We then asked whether NETs protection is correlated with anti-NETs antibody binding by analysing the NETs digestion and NETs staining pattern of individual SLE and ANA - sera. None of the ANA-ve serum (10\% diluted in PBS) bound to NETs while the majority of the SLE sera bound, indicating the presence of anti-NETs antibodies. Interestingly, sera from active SLE patients displayed significantly stronger binding to a decondensed area of NETs compared to inactive SLE serum at a similar level of anti-DNA antibody titre (Fig. 1b).

Next, we compared NETs protection between patients with active $(n=18)$ and inactive disease $(n=33)$. Serum (10\% neat sera diluted in PBS) from active SLE patients showed significantly higher NETs protection than serum from inactive patients in the presence of DNase (Fig. 1c). However, we did not find a significant correlation between the anti-dsDNA level and NETs protection or NET binding (Supplementary Fig. 1). During NETs formation, chromatin decondensation occurs and NETs are mainly composed of these relaxed decondensed $\mathrm{DNA}^{21,22}$. We then quantified the decondensed DNA staining of SLE patients sera according to the methods (doubleblinding scoring by two independent research scientists on a scale of 0-2). The decondensed DNA staining is also more predominant in active SLE patients (Fig. 1c).

\section{Anti-dsDNA antibodies are polyreactive}

In order to dissect the heterogeneity of the polyclonal set of anti-NETs antibodies in the serum from SLE patients, we cloned 206 humAbs from single plasmablasts $\left(\mathrm{CD}^{-}, \mathrm{CD} 20^{-}, \mathrm{CD} 19^{+}, \mathrm{CD} 27^{\mathrm{hi}}, \mathrm{CD} 38^{\mathrm{hi}}\right)$ sorted from PBMC of two active SLE patients with renal disease (Table 1, Supplementary Fig. 2). Following heavy and light chain PCR amplification antibodies were expressed and screened for ANA activity.

In all, 206 humAbs were screened for ANA, using an indirect immunofluorescence assay (IFA) on fixed Hep-2 cell. Forty-six ANA positive ENA negative humAbs were isolated, and their reactivity against histones, naked DNA and Crithidia kinetoplasts determined. Many humAbs have been found to be cross-reactive with both histones and DNA, a feature commonly observed in SLE patients who produce a high frequency of polyreactive and selfreactive mature naive $\mathrm{B}$ cell $^{23,24}$ (Fig. 2a). Furthermore, anti-dsDNA antibodies were shown to bind nonchromatin antigens including insulin, LPS, collagen, annexin II at high concentrations ${ }^{25-27}$. We assigned the 46 anti-ANA humAbs into four groups depending on their reactivity. Group A humAbs bound to all three antigens; histones, DNA and Crithidia and apoptotic cellls; group B bound to histones and DNA but not Crithidia, while group $C$ bound to histones only and group $D$ bound to DNA and Crithidia but not histones (Fig. 2a, b). Furthermore, 5/5 mAbs of group A bound to histone 1 while 2 group B mAbs bound to core histones (histone 2/ 3) (Supplementary Fig. 3). Interestingly, $4 / 5$ of the group A humAbs use VH3-23 while groups $B$ and $C$ have a more diverse $\mathrm{VH}$ usage (Supplementary Fig. 4d). The heavy chain CDR3 region of group A humAbs are more positively charged than the other three groups. The length of $\mathrm{CDR} 3$, the number of mutations and light chain usage is similar between the four groups (Supplementary Fig. 4). Finally, we showed than monoclonal dsDNA antibodies, which bound to Crithidia (marker for moderate to higher affinity DsDNA antibodies compared to ELISA) had significantly increased DNA decondensed scores (marker for decondensed area of NETs binding) (Fig. 2c).

\section{Group A mAbs bind and protect NET from nuclease digestion}

Next, we examined the binding of humAbs to neutrophils and NET; $5 / 5$ group A, $5 / 15$ group B and $4 / 21$ group $C$ antibodies $(10 \mu \mathrm{g} / \mathrm{ml})$ bound to both unstimulated neutrophils and NETs, while none of the group D antibodies $(10 \mu \mathrm{g} / \mathrm{ml})$ bound neutrophils or NETs (Fig. 3a). The group A and B mAbs bind to NETs at $0.1 \mu \mathrm{g} / \mathrm{ml}$ but did not bind to unstimulated neutrophil at $1 \mu \mathrm{g} / \mathrm{ml}$ (Supplementary Fig. 5B). Since the structure of NETs can vary with different stimuli, we examined the binding of monoclonal anti-dsDNA antibodies to NETs after stimulation with A23187 (divalent cation ionophore). Our results show a similar binding pattern to that observed with PMA stimulation (Supplementary Fig. 5A). However, the pattern of NETs staining differed between the humAbs from groups A, B \& C. Group A showed strong binding to the decondensed DNA area of the NETs stimulated by either PMA or A23187 (Fig. 3a), similar to the staining pattern of sera from active SLE patients, which also showed strong NETs protection activity (Fig. 1b). Groups B and C antibodies showed predominant binding to the condensed DNA area of the NET (Fig. 3a), while group D antibodies did not stain NETs.

We were next interested to see if any of the NETs binding antibodies could protect NET from nuclease digestion. Neutrophils were stimulated with PMA or calcium ionophore A23187 for $3 \mathrm{~h}$ and NETs structure was imaged by staining with sytox orange. NETs were preincubated with humAbs prior to the addition of Micrococcal nuclease or DNaseI $(1 \mathrm{U} / \mathrm{ml}$ MNase or DNase1 for $10 \mathrm{~min}$ at $37^{\circ} \mathrm{C}$ ) and the amount of digested DNA revealed by a fluorescent cytox orange assay. Group A antibodies $(n=5)$, which bound to decondensed DNA, 
A

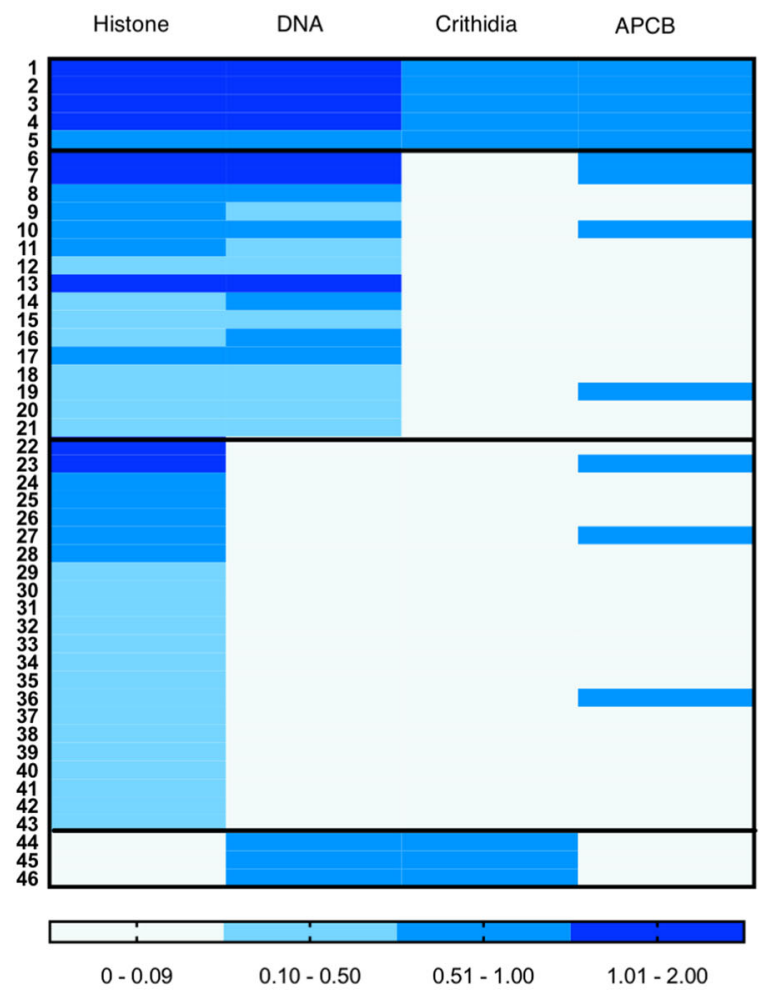

B

Group A
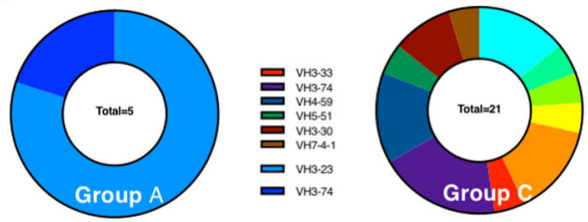

Group B
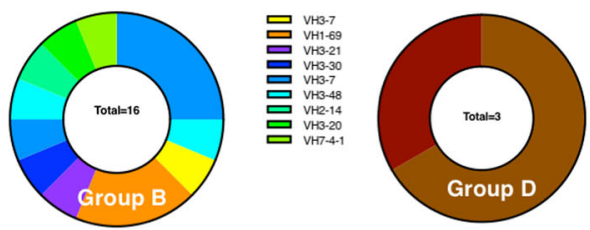

c

Group C

\section{Crithidia and NET binding}

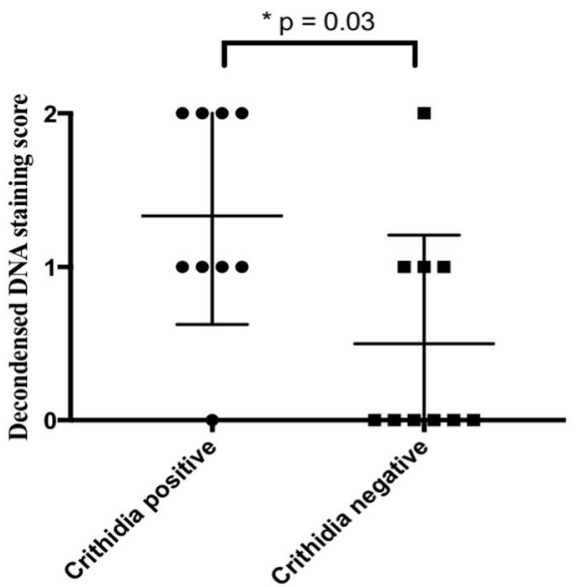

Fig. 2 IgH gene features and reactivity of four groups of humAbs. a Heat-map summarises the mAbs $(1 \mu \mathrm{g} / \mathrm{ml})$ reactivity against human histones, naked DNA (DNA) and Crithidia kinetoplast and UV treated/FAS ligand-induced apoptotic cells (APCB). Antibodies are grouped according to their reactivity. APCB and Crithidia results were scored as either 0 (negative) or 1 (positive). ELISA was repeated for three times and mean was calculated for the table, $n=3$. $\mathbf{b}$ Pie chart summarising antibody heavy chain gene family usage of each group of mAbs. Percentage of each gene family and the total number of mAbs were displayed. c The decondensed DNA binding score of the patients with positive crithidia staining or negative crithidia staining was compared. $N=18$, Student $t$-test, $p=0.03$.

showed strong NETs protection in a dose-dependent manner (from 1 to $100 \mu \mathrm{g} / \mathrm{ml}$ ) (Fig. 3b, c), similar to $10 \%$ sera from active SLE patients (Fig. 1c). HumAbs targeting condensed DNA $(n=11)$, from groups B, C and D, failed to show significant inhibition (Fig. 3b).

\section{Group A mAbs enhance type I IFN expression and NF-kB expression}

We then studied the effects of anti-NET humAbs on primary human monocytes and endothelial cells to determine mechanistic links between the impairment of NETs digestion and inflammatory immune responses in SLE. Monocytes were co-incubated with NETs coated with 12 humAbs from groups A-D (3 from each group) and induction of type I IFN was determined by RT-PCR.
Group A antibodies significantly enhanced type I IFN expression in monocytes compared to either the negative control antibody or the other 9 ANA mAbs (groups B, C and D) (Fig. 4a). Type I IFN enhancement is induced by group A mAbs $(n=3)$ in a dose-dependent manner (Supplementary Fig. 6A). We also tested the type I IFN expression induced by apoptotic cells co-incubated with 6 ApoC-binding humAbs from group $\mathrm{A}, \mathrm{B}$ and $\mathrm{C}$ as an apoptotic cell is the other important source of extracellular DNA in addition to NETs. However, we did not observe significantly enhanced or suppressed type I IFN expression $24 \mathrm{~h}$ after the co-incubation (Supplementary Fig. 6B)

To further investigate the mechanism of the type I IFN enhancement by NETs immune complexes, we mutated 

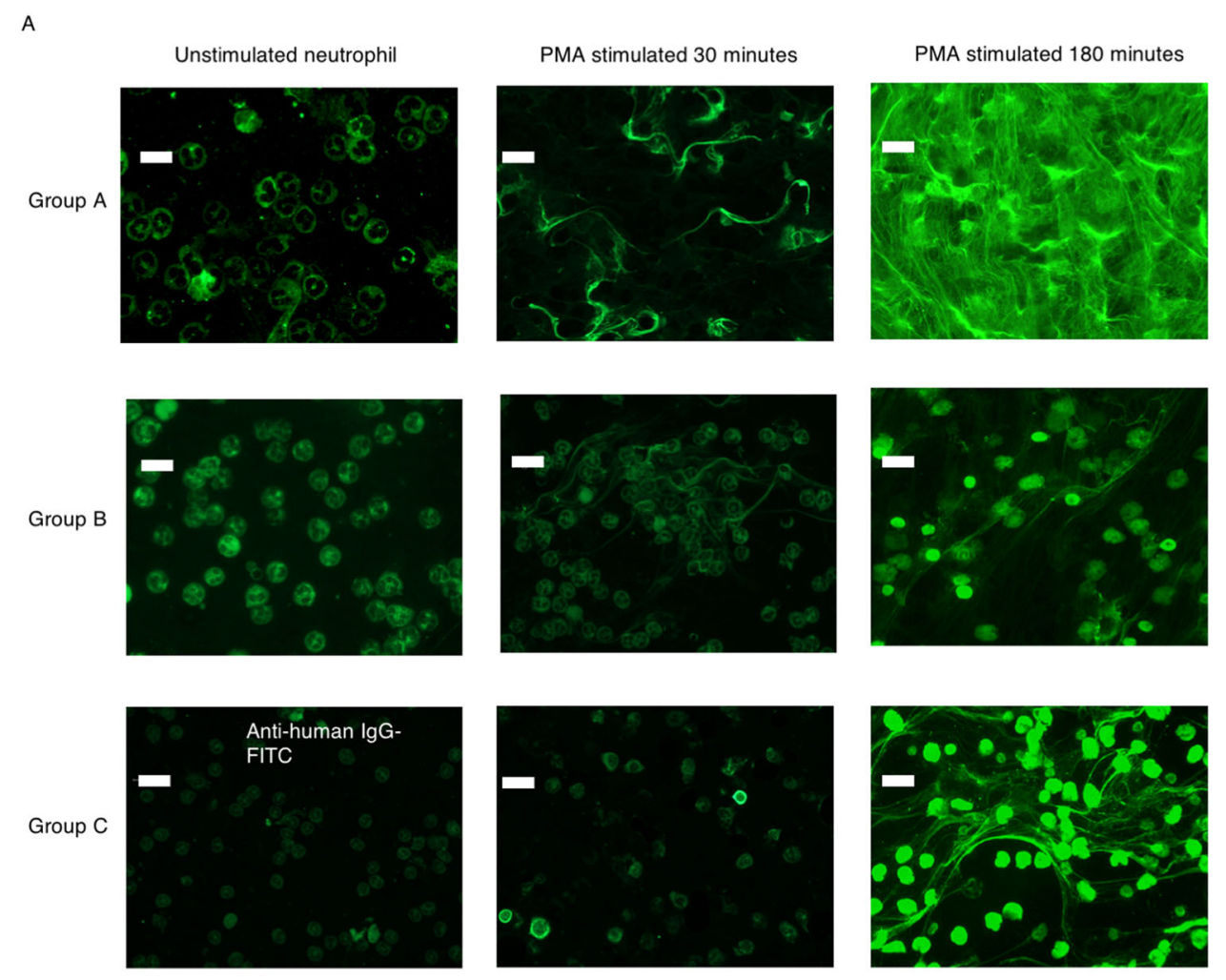

B

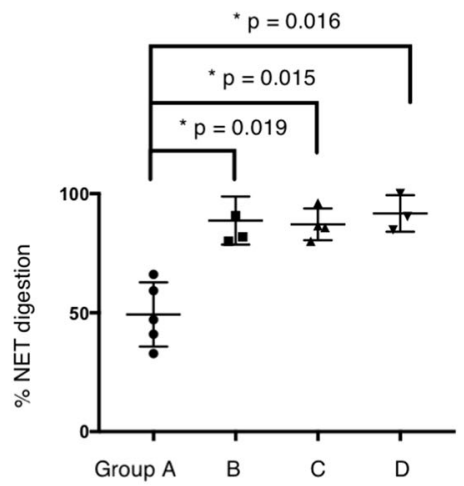

c

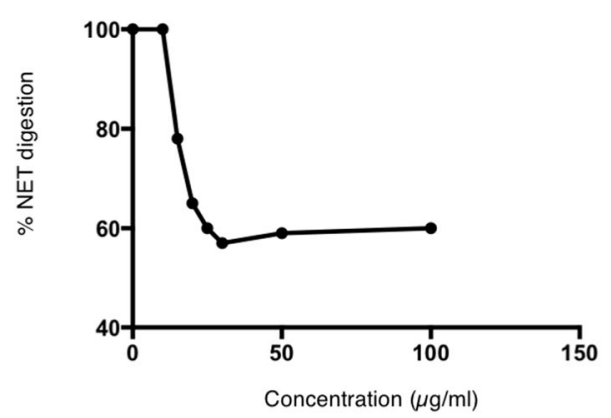

Fig. 3 Binding of humAbs to neutrophils and NET. a Representative mAb binding to neutrophils and NET from group A, B and C. Group D mAbs. The antibody binding to NET was detected by FITC-conjugated anti-human IgG while NET was visualised by DAPI, bar length $=25 \mu \mathrm{m}$. Neutrophils were either unstimulated or stimulated with $100 \mathrm{ng} / \mathrm{ml}$ PMA for 30 or $180 \mathrm{~min}$ before staining. Groups A and B bound to neutrophil cytoplasm at $10 \mu \mathrm{g} / \mathrm{ml}$ as shown in the figure but did not bind to unstimulated neutrophil cytoplasm at $1 \mu \mathrm{g} / \mathrm{ml}$. In total, 12/46 mAbs were positive for NET binding. $\mathbf{b}$ Percentage of NET digestion was measured in the presence of group A, B, C and D mAbs $(n=15)$ and displayed as a dot plot, repeated for three times. Data represent mean \pm SEM, Error bars indicate mean with $95 \% \mathrm{Cl}$ and Mann-Whitney $U$ was performed. c Group A mAbs protect NET from nuclease digestion in a dose-dependent manner, ranging from 1 to $100 \mu \mathrm{g} / \mathrm{ml}$. The graph is representative of one group A mAb (557A3), $n=$ 15.

the Fc portion of the heavy chain of group A mAb-557A3 by introducing the LALA mutation (mutations of leucine (L) to alanine (A) substitution at the position 234 and 235) known to restrict interaction with activating Fc-receptors. 557A3-LALA failed to enhance type I IFN when incubated with monocytes, suggesting that the process dependent on the uptake of antibody-NET complexes into the myeloid cells in an Fcg-R-dependent manner (Fig. 4b).

We then tested how anti-dsDNA antibody affects endothelial cells. Endothelial cells were coated with NET and humAbs for $24 \mathrm{~h}$ and amount of NET left on endothelial cells were visualised by DNA dye. NET-557A3 form stable immune complexes that stay on the 
A

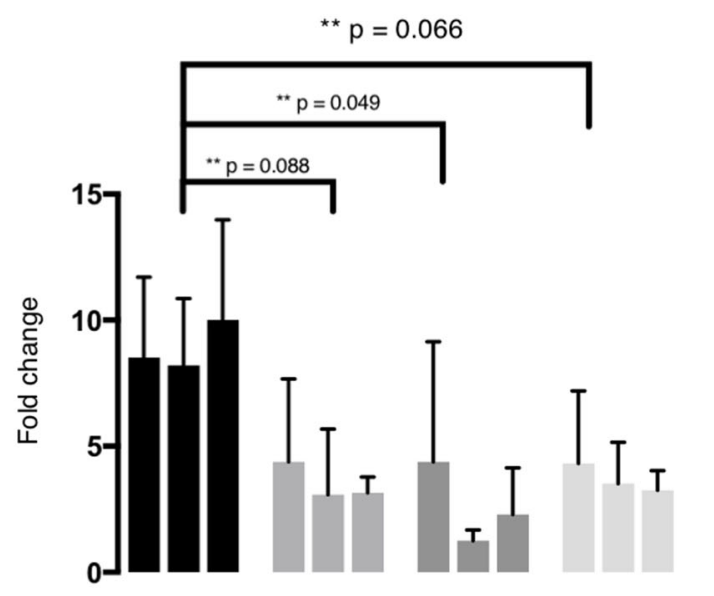

IFN $\beta$ expression

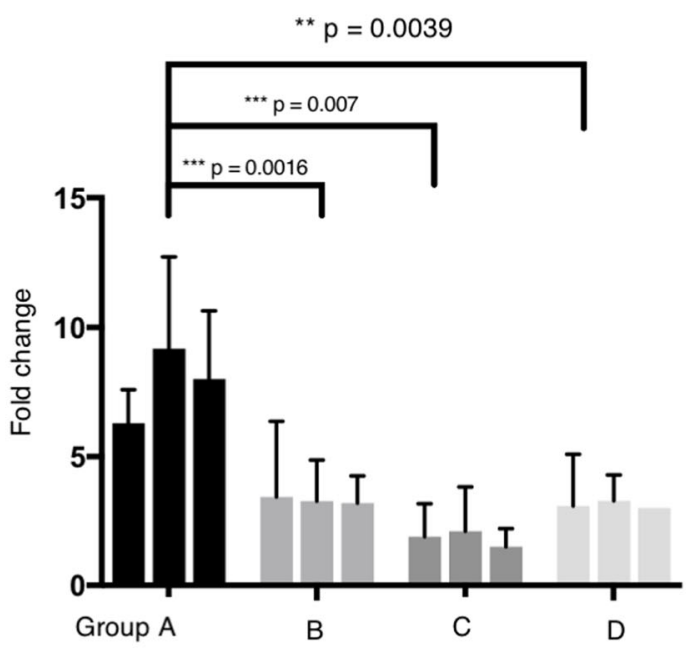

C

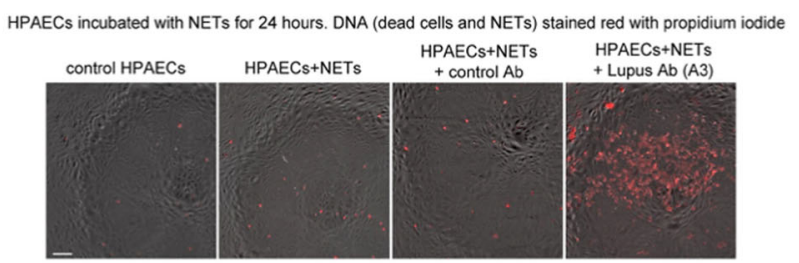

B

IFN $\alpha$ expression

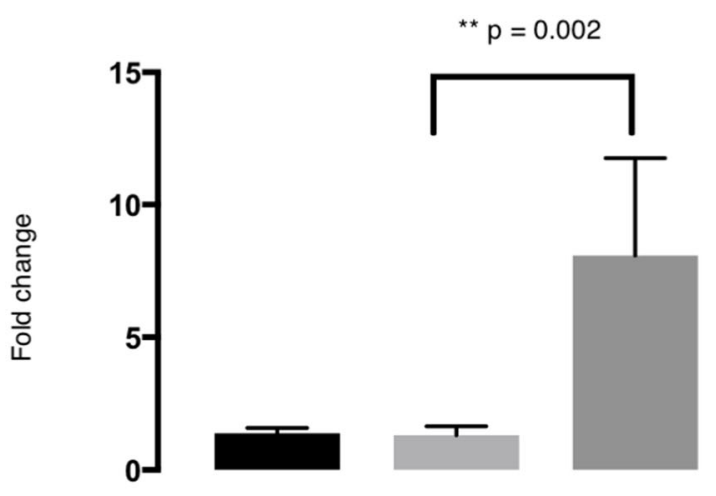

IFN $\beta$ expression

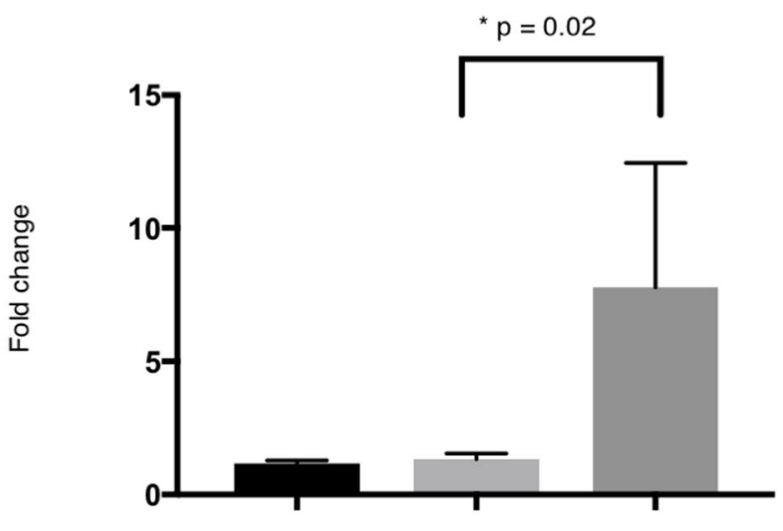

D

NFkB activity

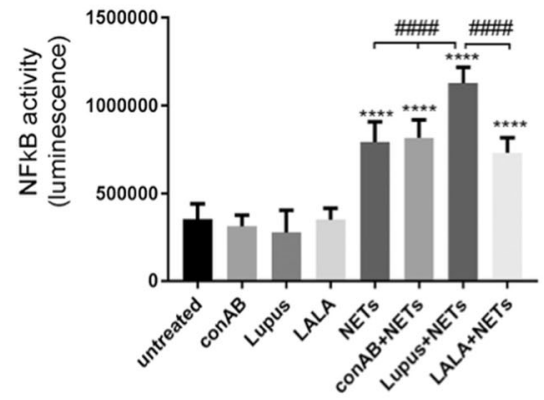

treatments

Fig. 4 Group A antibodies enhance type I IFN and NF-kB. a Monocytes were co-incubated with NET and three humAbs from each group $(n=12)$. Type I IFN expression was then measured by qPCR and fold change were calculated against negative control (anti-DENV antibody). Error bars indicate mean of three repeats with 95\% Cl, two-way ANOVA. b Type I IFN mRNA levels are measured by incubating NET, monocyte and group A humAb-557A3 (dark grey) or 557A3-LALA (light grey). Error bars indicate mean with $95 \% \mathrm{Cl}$ Student $t$-test, $n=4$. c Significantly more NET stayed on HPAECs after $24 \mathrm{~h}$ when coincubated with group A humAb-557A3. HPAECs were incubated with NETs for $24 \mathrm{~h}$ in the presence of control and $557 \mathrm{~A} 3$ before staining with propidium iodide. The addition of group A antibody markedly attenuated degradation of NETs (red, last image on the right). Bar $=50 \mu \mathrm{m}$. $\mathbf{d}$ HPAECs infected with AdNFkB-luc were incubated with NETs in the presence or absence of group A antibody 557A3. Following overnight incubation, NF-kB activity was measured in a luciferase reporter assay. ${ }^{* * *} P<0.0001$, comparisons with untreated control; ${ }^{\# \# \#} P<0.0001$, comparisons, as indicated. ANOVA with Tukey post-test, $n=4$. 
endothelial cells after $24 \mathrm{~h}$ while NET alone or with group $\mathrm{B}$ antibodies were digested (Fig. 4c) It has been suggested that undigested NET may directly damage endothelial cells REF, so we next tested the ability of NET-humAb immune complexes to activate NF- $\mathrm{KB}$ in endothelial cells. NET-humAb immune complexes stimulated NF- $k B$ activity in endothelial cells twofold compared to a control IgG1 anti-dengue humAb (Fig. 4d). HumAb-557A3-NET immune complex enhancement of NF- $\mathrm{kB}$ activity in endothelial cells was also Fcg-R dependent as the activity was lost when the LALA version of HumAb-557A3 was used (Fig. 4d).

\section{Group A mAbs deposit in nephritic kidney}

We were interested to determine whether the antiNETs humAb would interact with inflamed tissue in vivo. We used an active autoimmune nephrotoxic nephritis (NTN) model that has predominant kidney inflammation driven by immune complexes deposition, mimicking lupus nephritis. To this end, we injected intraperitoneally $100 \mu \mathrm{g}$ of representative humAbs from groups A-D $(n=$ 8) into mice primed for immune complex-mediated nephritis (as described in "Materials and methods" section) at day 4. The concentration of circulating humAb was measured 2 and $6 \mathrm{~h}$ following injection. Group A humAb-557A3 and 157 B9 deposited exclusively in glomerular areas (Fig. 5a, c) and was completely cleared from the serum $6 \mathrm{~h}$ after injection (Fig. $5 \mathrm{~b}$ ). The groups B, C and D humAbs $(n=6)$ that do not protect NET did not deposit in the kidney and they remained in circulation after $6 \mathrm{~h}$. None of the anti-NET antibody deposits in a healthy kidney from untreated wild-type mice.

\section{Discussion}

Our study revealed a previously unknown subset of pathogenic anti-dsDNA antibody that enhances the inflammation in SLE. Our study also reveals a novel pathogenic mechanism in SLE, that anti-dsDNA humAbs stabilise NETs and enhance inflammation. Using single plasma cell expression cloning, we identified a subset of human monoclonal anti-dsDNA antibodies, characterised by binding to decondensed chromatin in NETs which localises to the damaged kidney in vivo and promotes the expression of IFN- $\alpha$ by monocytes in an FcR-dependent manner. Our results encapsulate two main features of nephritogenic antibodies; deposition within the kidney and activation of inflammatory immune responses by inhibiting NET digestion.

Our study for the first time identifies that anti-dsDNA antibodies that bind to both histones and Crithidia DNA prevent NET from nuclease digestion. Although the clinical significance of anti-dsDNA antibodies is well known, there are still significant gaps in our understanding as to how they are involved in disease progression. Analysis of murine monoclonal antibodies shows that only some anti-dsDNA antibodies cause tissue damage and lupus nephritis ${ }^{5}$. Level of anti-dsDNA antibodies also does not correlate with disease activity; some individuals have a high titre of anti-dsDNA antibodies and remain well ${ }^{1}$. These findings indicate that anti-dsDNA antibodies are not all alike and the antigen-binding specificities may determine their pathogenicity ${ }^{1,28}$. Histone ELISA and dsDNA ELISA were selected because DNA, histone and DNA-histone complexes are the main component of the nucleosome in vivo, and anti-chromatin/ nucleosome antibodies were found to be more correlated with disease activity ${ }^{29-31}$. Crithidia luciliae test was also used for the characterisation because it has a higher specificity for SLE as it only detects anti-dsDNA antibodies while ELISA detects both anti-ssDNA and antidsDNA antibodies ${ }^{32}$.

Previous studies found that some acute SLE patients have clonal expanded autoreactive $\mathrm{VH} 4-34^{+}$B cells, which produce polyreactive antibodies (9G4 antibodies) that react with dsDNA, histone, chromatin and apoptotic cells $^{33}$. The 9G4 antibodies are correlated with disease activity, and clinical manifestations but the pathogenic function of them in comparison with anti-dsDNA antibody, in general, is still unexplored ${ }^{4,33}$. In this study, we observed clonal expansion of $\mathrm{VH} 3-23^{+}$plasmablasts in group A antibodies, which are also polyreactive to dsDNA, histone and apoptotic cells. However, we did not find any VH4-34 antibodies from the two acute SLE patients in our study, which suggests that additional autoreactive VH antibody-secreting cells (ASCs) expansion may also be correlated with disease activity. We also found that group A humAbs have significantly higher CDR3 charge, which was suggested to contribute to the anti-dsDNA antibody autoreactivity ${ }^{33-35}$.

Retrospective analysis of autoantibody and inflammatory mediators in individuals who progress to SLE show anti-chromatin antibodies precede the simultaneous increase in IFN- $\alpha$ levels and detection of anti-dsDNA antibodies $^{1,36}$. We extend these finding by showing that anti-dsDNA antibodies, which bind to decondensed chromatin within NETs and inhibit their breakdown. These NETs protective mAbs bind to histone 1 and Crithidia, which might cover the chromatin, thereby possibly prevent nuclease from accessing the cleavage sites. The group A mAbs also activate monocytes and endothelial cells and deposited in the kidneys of nephritis mice. In this study all anti-dsDNA antibodies which inhibited NETs degradation bound to the Crithidia DNA and histone 1 linker protein, which connects core nucleosome subunits and has a role in the formation of higher-order chromatin structures ${ }^{37-39}$. Unfoldment of compact chromatin structure in NETs may promote the formation of histone 1 antibodies resulting in stabilisation of anti-NETs 


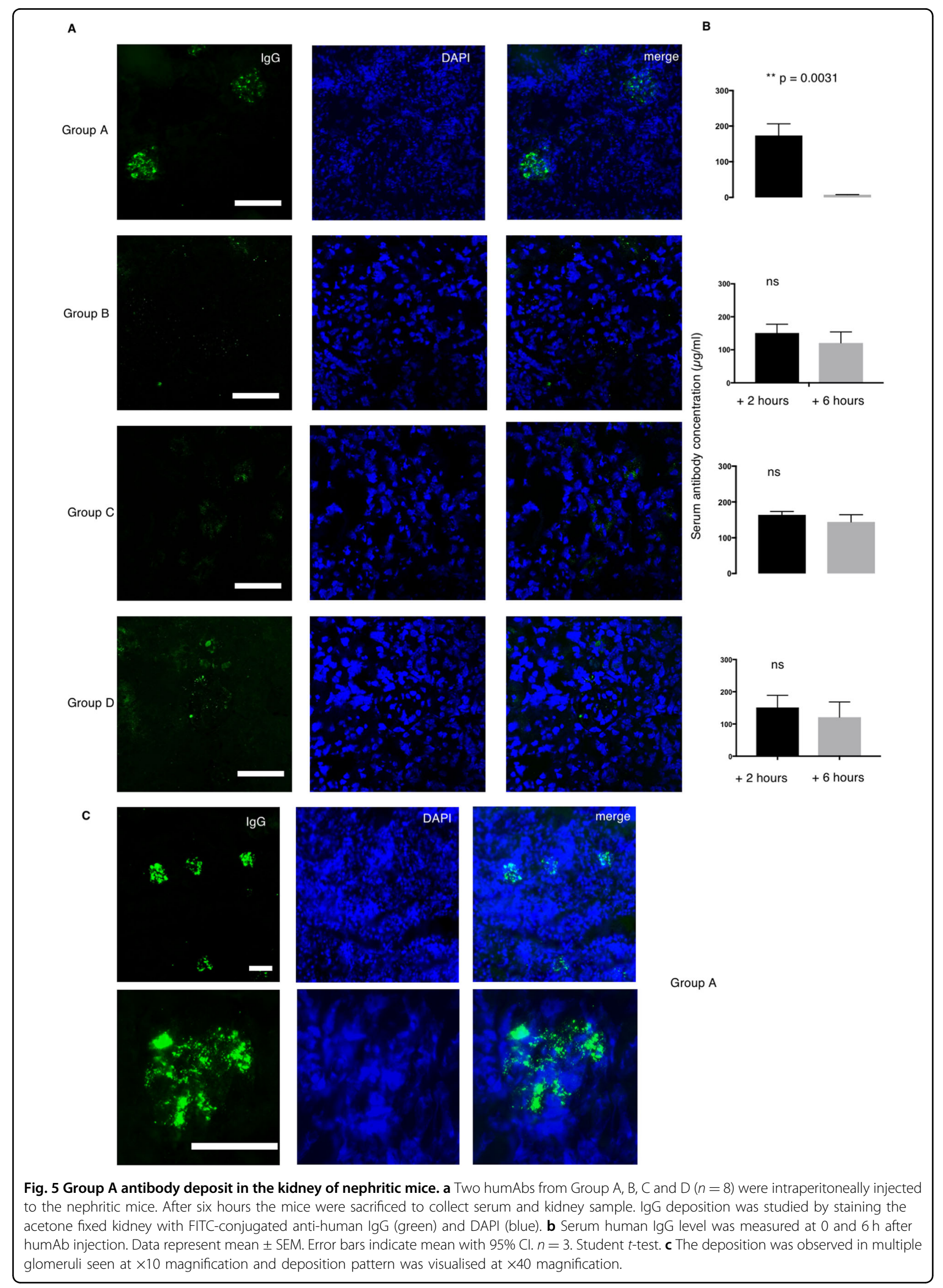

Official journal of the Cell Death Differentiation Association 


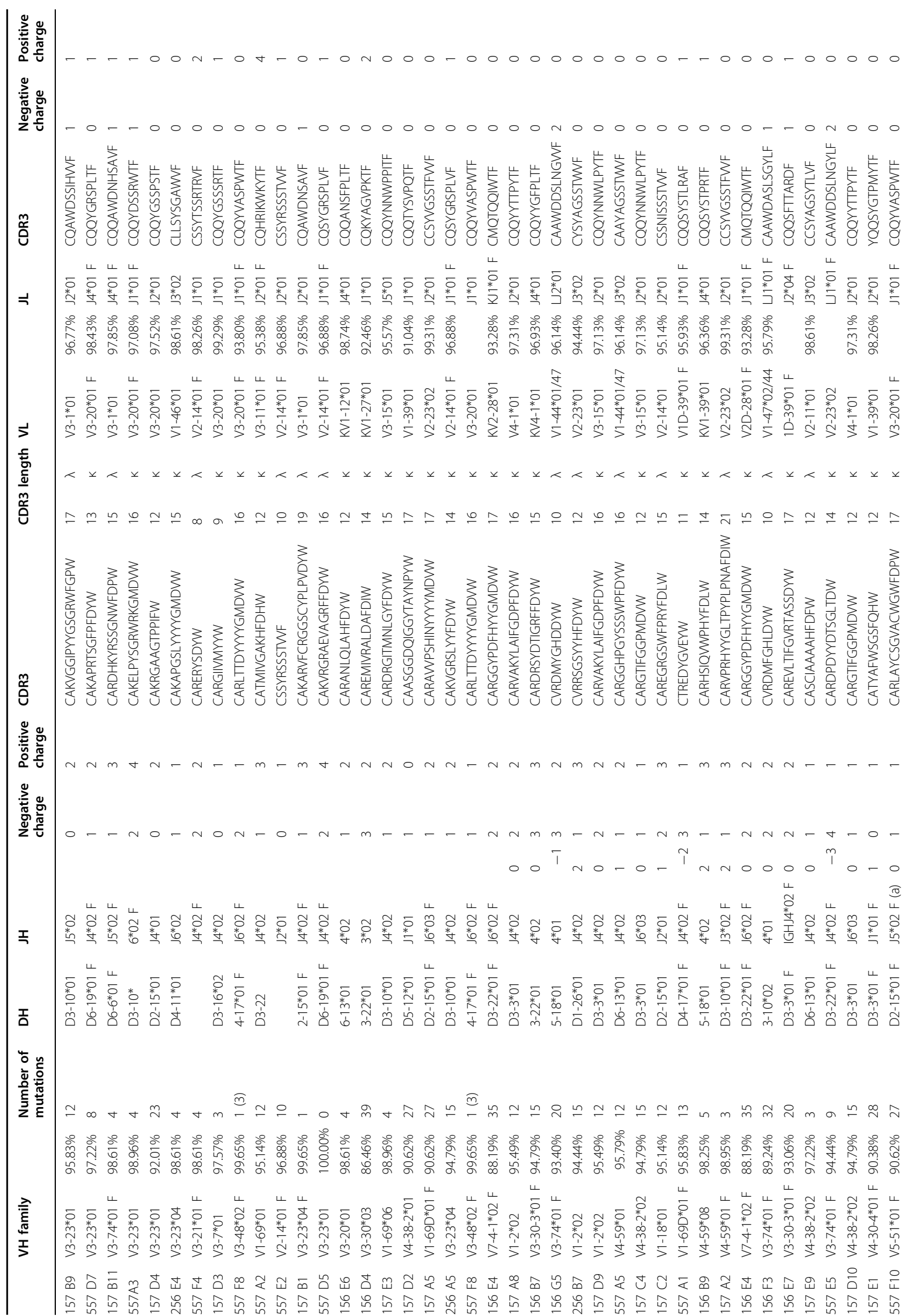




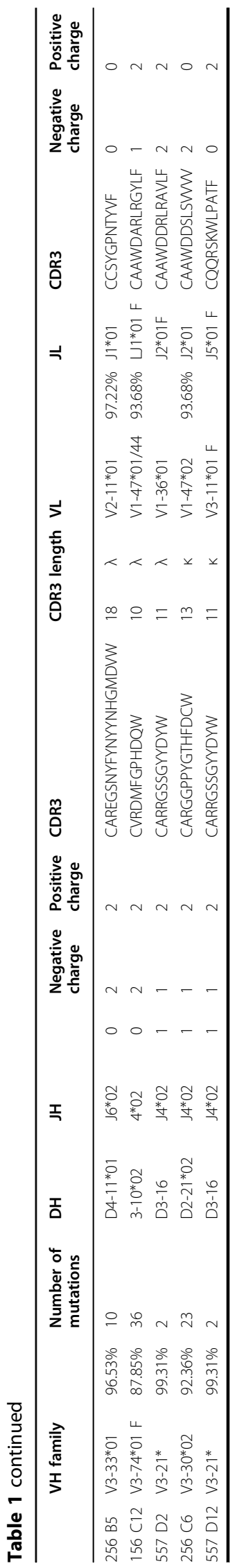

antibody immune complexes and subsequent proinflammatory immune responses ${ }^{13}$. In addition, histone 1 is also a component of apoptotic chromatin constituents in glomerular basement dense electron deposits that are known to be targets of nephritogenic anti-DNA antibodies ${ }^{5,10,40,41}$. Previous studies have linked polyclonal anti-histone 1 with SLE disease activity $^{29,31}$; however, our data showed that these antibodies can lead to inflammatory immune responses and endothelial cell activation.

Our study revealed that only this subset of crossreactive anti-dsDNA antibodies could enhance inflammatory response. NETs is secreted by neutrophils by an active process called NETosis that sometimes trigger cell death $^{22,42}$. NET is composed of chromatin and neutrophil granular proteins that is suggested to capture and neutralise pathogens ${ }^{13}$. However, NET was also found to promote an inflammatory response in SLE by stimulating immune cells or endothelial cells ${ }^{13,43,44}$. Previous studies revealed that undigested NETs promotes IFN- $\alpha$ secretion by plasmacytoid dendritic cells $^{10,12,15,18,45,46}$. Monocytes, tissue macrophages and endothelial cells are believed to be the main site for immune complex and extracellular DNA clearance but do not secrete pro-inflammatory cytokines following NET elimination in healthy controls ${ }^{47}$. By contrast, incubation of monocyte with NETs isolated from patients with SLE can also activate cGAS and caspase 1 mediated inflammatory response, suggesting that certain forms of NETs are immunostimulatory ${ }^{45,48}$. In addition, the dsDNA complex in NETs was shown to induce type I IFN production from $\mathrm{pDC}$ in a TLR9 dependent manner, driven by the TLR adaptor MydD88 and IRF $^{49}$. Only the NETs dsDNA-protein complex but not naked DNA is able to activate TLR9 in pDC due to the stability of the complex. Furthermore, oxidised DNA from mitochondria is also found to activate cGAS intracellularly while the NETs associated proteins were shown to engage NLRP3 inflammasome, leading to IL-1 and IL-18 release ${ }^{45,50}$. Here we show that stabilisation of NETs by dsDNA antibodies also stimulates the expression of IFN- $\alpha$ by peripheral blood monocytes, NF-kB activity in endothelial cells, illustrating an alternative inflammatory pathway triggered by NETs. We also demonstrated that the anti-dsDNA antibody - NETs complex stimulate the monocyte and endothelial cells in an FcR-dependent manner. However, it should be noted that the structure of NETs stimulated by different stimuli would have a distinct structure in vivo, therefore our finding may only represent some of the NETs structure. Binding of IgG-containing immune complexes to Fc-receptors is a crucial step in the development of lupus nephritis. In murine models of SLE, activation of Fc-receptors on circulating white cells 
rather than renal resident cells is responsible for inflammatory immune responses and tissue damage ${ }^{51,52}$. Data from experimental models of SLE and clinical renal biopsies show a striking association between markers of $\mathrm{Fc} \gamma \mathrm{R}$ mediated monocyte activation and renal inflammatory immune responses in SLE, highlighting the clinical relevance of NET induced monocyte activation ${ }^{52-54}$. The inflammatory immune response is likely to involve several diffident factors including immune complexes, complement activation, recruitment of inflammatory leukocytes and cytokine signalling. Our findings link anti-dsDNA/ NET immune complex formation to several aspects of inflammatory changes causing tissue damage in LN and highlight contribution of other innate immune cells (neutrophils and monocytes) besides plasmacytoid dendritic cells in the pathogenesis of SLE.

We then exhibit that the potentially pathogenic antidsDNA mAbs may amplify the inflammation instead of initiating it. We demonstrate that the anti-dsDNA mAbs alone does not enhance inflammatory response when coincubated with monocyte or endothelial cells. Furthermore, the anti-dsDNA antibodies are only localised to kidneys of nephritic mice and not to healthy controls, suggesting that monoclonal antibodies identified in this study are more likely to exacerbate inflammatory immune responses and tissue damage but not to initiate $i^{55-57}$. Rising evidence suggesting that both anti-dsDNA antibodies and NET are more likely to amplify rather than directly cause tissue pathology 6,12,46,48,49,57-59. AntidsDNA antibodies deposit in the kidney of lupus nephritis patients but the mechanism is still controversial $^{5,60,61}$. The group A mAbs from our study rapidly bind to glomerulus of nephritic mice but not to the healthy controls, indicating that they may recognise antigens exposed during inflammation. Therefore, they probably recognise antigens that are exposed during kidney inflammation but not native glomerulus antigens as found by other groups ${ }^{25,62,63}$. During inflammation, increased number of cells undergo apoptosis or even secondary necrosis, leading to the release of intracellular contents including nucleosome. These nucleosomes may serve as the antigen target for the anti-dsDNA antibodies to deposit in the kidney ${ }^{38,40,64}$. Experimental data from murine models indicates that multiple SLE autoantibody can trigger LN and factors such as Ig isotype, subclass, charge avidity, murine strain and other factors can have a role in promoting disease $\mathrm{e}^{2,5,26,65}$.

Evidence from murine models of SLE suggests that chromatin-containing microparticles are responsible for the initial loss of tolerance to nuclear constituents and can prime neutrophils for NETosis ${ }^{27,66-71}$. Netting neutrophils leads to endothelial activation and further release of chromatin-containing microparticles, resulting in perpetuating the cycle of inflammatory immune responses, accelerated atherosclerosis, thrombosis and tissue damage in the kidneys, skin and blood ${ }^{10,12,45,72-74}$. The pathogenic anti-dsDNA antibodies may then bind and stabilise the NETs, further stimulate the inflammatory response and tissue damage according to our findings. We, therefore, extend the current understanding of anti-dsDNA antibody-mediated inflammation during the course of SLE.

The study does have a number of limitations. The SLE disease scores were not based solely on LN activity but did incorporate other disease domains such as constitutional, musculoskeletal and cutaneous symptoms, however DsDNA antibodies are also associated with progression skin in SLE. It will also be important to determine the impact of nephritogenic anti-dsDNA antibodies using BLIAG2004 scoring criteria on objective measures of renal disease in prospective longitudinal studies in larger SLE cohorts. Further studies will be required to assess what component of the glomerular basement membrane do the group A anti-dsDNA antibodies bind, and the influence of IgG subclass on NETs stabilisation, and endothelial activation. As we have only identified few Group A antibodies further studies with larger numbers will be necessary to further clarify the function of the subsets of anti-dsDNA antibodies in LN. It should also be noted that the capacity of group A antibodies to bind to apoptotic blebs and its influence on the progression of $\mathrm{LN}$ will need further study. Our data offer an explanation, for the discordance between anti-dsDNA antibody concentration and SLE activity and suggests the potential of an alternative diagnostic test to monitor SLE activity, which will need to be studied, longitudinally in larger SLE disease cohorts with appropriate disease controls. As we have demonstrated the correlation between Crithidia staining, decondensed NET binding and active SLE, it is plausible to use Crithidia and decondensed NET binding for all the SLE patients as a biomarker for precision therapy. Treatments to accelerate NETs breakdown in SLE patients with strong decondensed NET binding could be considered in the future.

\section{Study approval}

The study is approved by Imperial College Healthcare, Department of Surgery and Cancer under the ICHTB HTA licence: 12275, REC Wales approval: 12/WA/0196.

\footnotetext{
Acknowledgements

We thank Wellcome Trust and MRC Imperial College ICIC for funding the project, Prof Marina Botto for her kind advice, and consented patients for their participation.

\section{Author details}

'Division of Immunology and Inflammation, Department of Medicine, Hammersmith Campus, Imperial College London, London W12 ONN, UK. Department of Medicine, Centre for Immunology \& Vaccinology, Chelsea and Westminster Hospital, Imperial College London, London SW10 9NH, UK.

${ }^{3}$ Department of Medicine, Centre for Pharmacology and Therapeutics, Imperial College London, London W12 OHS, UK. ${ }^{4}$ Division of Immunology and
} 
Inflammation, Centre for Complement and Inflammation Research, Imperial College London, London W12 ONN, UK. ${ }^{5}$ Department of Infection and Immunity, Charing Cross Hospital North West London Pathology, London W6 8RF, UK. 'Division of Medical Sciences, John Radcliffe Hospital, Oxford University, Oxford OX3 9DU, UK

\section{Conflict of interest}

The authors declare that they have no conflict of interest.

\section{Publisher's note}

Springer Nature remains neutral with regard to jurisdictional claims in published maps and institutional affiliations.

Supplementary Information accompanies this paper at (https://doi.org/ 10.1038/s41419-020-02928-6).

Received: 17 February 2020 Revised: 7 August 2020 Accepted: 24 August 2020

Published online: 09 September 2020

\section{References}

1. Pisetsky, D. S. Anti-DNA antibodies-quintessential biomarkers of SLE. Nat. Rev. Rheumatol. 12, 102-110 (2016).

2. Rekvig, O. P., Kalaaji, M. \& Nossent, H. Anti-DNA antibody subpopulations and lupus nephritis. Autoimmun. Rev. 3, 1-6 (2004).

3. Hahn, B. H. Antibodies to DNA. N. Engl. J. Med 338, 1359-1368 (1998).

4. Tipton, C. M. et al. Diversity, cellular origin and autoreactivity of antibodysecreting cell population expansions in acute systemic lupus erythematosus. Nat. Immunol. 16, 755-765 (2015).

5. Ehrenstein, M. R. et al. Human lgG anti-DNA antibodies deposit in kidneys and induce proteinuria in SCID mice. Kidney Int. 48, 705-711 (1995).

6. Henault, J. et al. Self-reactive IgE exacerbates interferon responses associated with autoimmunity. Nat. Immunol. 17, 196-203 (2016).

7. Schur, P. H. \& Sandson, J. Immunologic factors and clinical activity in systemic lupus erythematosus. N. Engl. J. Med. 278, 533-538 (1968).

8. Henault, J. et al. Noncanonical autophagy is required for type I interferon secretion in response to DNA-immune complexes. Immunity 37, 986-997 (2012).

9. Leadbetter, E. A. et al. Chromatin-lgG complexes activate B cells by dual engagement of lgM and Toll-like receptors. Nature 416, 603-607 (2002).

10. Hakkim, A. et al. Impairment of neutrophil extracellular trap degradation is associated with lupus nephritis. Proc. Natl Acad. Sci. USA 107, 9813-9818 (2010).

11. Tsokos, G. C., Lo, M. S., Reis, P. C. \& Sullivan, K. E. New insights into the immunopathogenesis of systemic lupus erythematosus. Nat. rev. Rheumatol. 12, 716-730 (2016).

12. Garcia-Romo, G. S. et al. Netting neutrophils are major inducers of type I IFN production in pediatric systemic lupus erythematosus. Sci. Transl. Med. 3, $73 r a 20$ (2011).

13. Brinkmann, V. et al. Neutrophil extracellular traps kill bacteria. Science $\mathbf{3 0 3}$, 1532-1535 (2004)

14. Branzk, N. et al. Neutrophils sense microbe size and selectively release neutrophil extracellular traps in response to large pathogens. Nat. Immunol. 15 1017-1025 (2014).

15. Kessenbrock, K. et al. Netting neutrophils in autoimmune small-vessel vasculitis. Nat. Med. 15, 623-625 (2009)

16. Grieves, J. L. et al. Exonuclease TREX1 degrades double-stranded DNA to prevent spontaneous lupus-like inflammatory disease. Proc. Natl Acad. Sci. USA 112, 5117-5122 (2015).

17. Leffler, J. et al. A subset of patients with systemic lupus erythematosus fails to degrade DNA from multiple clinically relevant sources. Arthritis Res. Ther. 17, 205 (2015).

18. Leffler, J. et al. Neutrophil extracellular traps that are not degraded in systemic lupus erythematosus activate complement exacerbating the disease. J. Immunol. 188, 3522-3531 (2012).

19. Brinkmann, V. \& Zychlinsky, A. Neutrophil extracellular traps: is immunity the second function of chromatin? J. Cell Biol. 198, 773-783 (2012).
20. Wang, Y. et al. Histone hypercitrullination mediates chromatin decondensation and neutrophil extracellular trap formation. J. Cell Biol. 184, 205-213 (2009).

21. Ermert, D. et al. Mouse neutrophil extracellular traps in microbial infections. J. Innate Immun. 1, 181-193 (2009).

22. Papayannopoulos, V., Metzler, K. D., Hakkim, A. \& Zychlinsky, A. Neutrophil elastase and myeloperoxidase regulate the formation of neutrophil extracellular traps. J. Cell Biol. 191, 677-691 (2010).

23. Wardemann, H. et al. Predominant autoantibody production by early human B cell precursors. Science 301, 1374-1377 (2003).

24. Mietzner, B. et al. Autoreactive lgG memory antibodies in patients with systemic lupus erythematosus arise from nonreactive and polyreactive precursors. Proc. Natl Acad. Sci. USA 105, 9727-9732 (2008).

25. Yung, S., Cheung, K. F., Zhang, Q. \& Chan, T. M. Anti-dsDNA antibodies bind to mesangial annexin II in lupus nephritis. J. Am. Soc. Nephrol. 21, 1912-1927 (2010).

26. Krishnan, M. R., Wang, C. \& Marion, T. N. Anti-DNA autoantibodies initiate experimental lupus nephritis by binding directly to the glomerular basement membrane in mice. Kidney Int. 82, 184-192 (2012).

27. Yurasov, S. et al. Defective B cell tolerance checkpoints in systemic lupus erythematosus. J. Exp. Med. 201, 703-711 (2005).

28. Pisetsky, D. S. The origin and properties of extracellular DNA: from PAMP to DAMP. Clin. Immunol. 144, 32-40 (2012).

29. Sun, X. Y., Shi, J., Han, L., Su, Y. \& Li, Z. G. Anti-histones antibodies in systemic lupus erythematosus: prevalence and frequency in neuropsychiatric lupus. J. Clin. Lab Anal. 22, 271-277 (2008).

30. Cortes-Hernandez, J. et al. Antihistone and anti-double-stranded deoxyribonucleic acid antibodies are associated with renal disease in systemic lupus erythematosus. Am. J. Med. 116, 165-173 (2004).

31. Schett, $\mathrm{G}$. et al. The autoimmune response to chromatin antigens in systemic lupus erythematosus: autoantibodies against histone $\mathrm{H} 1$ are a highly specific marker for SLE associated with increased disease activity. Lupus 11, 704-715 (2002).

32. Conrad, K. et al. High sensitive detection of double-stranded DNA autoantibodies by a modified Crithidia luciliae immunofluorescence test. Ann. N. Y. Acad. Sci. 1173, 180-185 (2009).

33. Richardson, C. et al. Molecular basis of $9 \mathrm{G} 4 \mathrm{~B}$ cell autoreactivity in human systemic lupus erythematosus. J. Immunol. 191, 4926-4939 (2013).

34. Li, Z., Schettino, E. W., Padlan, E. A., Ikematsu, H. \& Casali, P. Structure-function analysis of a lupus anti-DNA autoantibody: central role of the heavy chain complementarity-determining region $3 \mathrm{Arg}$ in binding of double- and singlestranded DNA. Eur. J. Immunol. 30, 2015-2026 (2000).

35. Wellmann, $U$. et al. The evolution of human anti-double-stranded DNA autoantibodies. Proc. Natl Acad. Sci. USA 102, 9258-9263 (2005).

36. Steiman, A. J. et al. Anti-dsDNA and antichromatin antibody isotypes in serologically active clinically quiescent systemic lupus erythematosus. J. Rheumatol. 42, 810-816 (2015).

37. Luger, K., Dechassa, M. L. \& Tremethick, D. J. New insights into nucleosome and chromatin structure: an ordered state or a disordered affair? Nat. Rev. Mol. Cell Biol. 13, 436-447 (2012).

38. Fenton, K. A., Tommeras, B., Marion, T. N. \& Rekvig, O. P. Pure anti-dsDNA mAbs need chromatin structures to promote glomerular mesangial deposits in BALB/C mice. Autoimmunity 43, 179-188 (2010).

39. Happel, N. \& Doenecke, D. Histone $\mathrm{H} 1$ and its isoforms: contribution to chromatin structure and function. Gene 431, 1-12 (2009).

40. Kalaaji, M. et al. Glomerular apoptotic nucleosomes are central target structures for nephritogenic antibodies in human SLE nephritis. Kidney Int. 71, 664-672 (2007)

41. Hedberg, A., Fismen, S., Fenton, K. A., Mortensen, E. S. \& Rekvig, O. P. Deposition of chromatin-lgG complexes in skin of nephritic MRL-lpr/lpr mice is associated with increased local matrix metalloprotease activities. Exp. Dermatol. 19, e265-e274 (2010).

42. Metzler, K. D. et al. Myeloperoxidase is required for neutrophil extracellular trap formation: implications for innate immunity. Blood 117, 953-959 (2011).

43. Kaplan, M. J. \& Radic, M. Neutrophil extracellular traps: double-edged swords of innate immunity. J. Immunol. 189, 2689-2695 (2012).

44. Kaplan, M. J. Neutrophils in the pathogenesis and manifestations of SLE. Nat. Rev. Rheumatol. 7, 691-699 (2011).

45. Kahlenberg, J. M., Carmona-Rivera, C., Smith, C. K. \& Kaplan, M. J. Neutrophil extracellular Trap-associated protein activation of the NLRP3 inflammasome is enhanced in lupus macrophages. J. Immunol. 190, 1217-1226 (2013). 
46. Lood, C. et al. Neutrophil extracellular traps enriched in oxidized mitochondrial DNA are interferogenic and contribute to lupus-like disease. Nat. Med. 22, 146-153 (2016).

47. Farrera, C. \& Fadeel, B. Macrophage clearance of neutrophil extracellular traps is a silent process. J. Immunol. 191, 2647-2656 (2013).

48. Caielli, S. et al. Oxidized mitochondrial nucleoids released by neutrophils drive type I interferon production in human lupus. J. Exp. Med. 213, 697-713 (2016).

49. Lande, R. et al. Neutrophils activate plasmacytoid dendritic cells by releasing self-DNA-peptide complexes in systemic lupus erythematosus. Sci. Transl. Med. 3, 73 ra19 (2011)

50. Miller, L. S. et al. Inflammasome-mediated production of IL-1 beta is required for neutrophil recruitment against Staphylococcus aureus in vivo. J. Immunol. 179, 6933-6942 (2007).

51. Asselin-Paturel, C. et al. Mouse type I IFN-producing cells are immature APCs with plasmacytoid morphology. Nat. Immunol. 2, 1144-1150 (2001).

52. Bergtold, A., Gavhane, A., D'Agati, V., Madaio, M. \& Clynes, R. FcR-bearing myeloid cells are responsible for triggering murine lupus nephritis. J. Immunol. 177, 7287-7295 (2006)

53. Peterson, K. S. et al. Characterization of heterogeneity in the molecular pathogenesis of lupus nephritis from transcriptional profiles of laser-captured glomeruli. J. Clin. Invest. 113, 1722-1733 (2004).

54. Katsiari, C. G., Liossis, S. N. \& Sfikakis, P. P. The pathophysiologic role of monocytes and macrophages in systemic lupus erythematosus: a reappraisal. Semin. Arthritis Rheum. 39, 491-503 (2010).

55. Arbuckle, M. R. et al. Development of autoantibodies before the clinical onset of systemic lupus erythematosus. N. Engl. J. Med. 349, 1526-1533 (2003).

56. Dieker, J. et al. Circulating apoptotic microparticles in systemic lupus erythematosus patients drive the activation of dendritic cell subsets and prime neutrophils for NETosis. Arthritis Rheumatol. 68, 462-472 (2016).

57. Fu, S. M., Dai, C., Zhao, Z. \& Gaskin, F. Anti-dsDNA antibodies are one of the many autoantibodies in systemic lupus erythematosus. F1000Res 4, 939 (2015).

58. Wang, H. T., Li, T., Chen, S., Gu, Y. Y. \& Ye, S. Neutrophil extracellular trap mitochondrial DNA and its autoantibody in systemic lupus erythematosus and a proof-of-concept trial of metformin. Arthritis Rheumatol. 67, 3190-3200 (2015).

59. Soni, C. \& Reizis, B. DNA as a self-antigen: nature and regulation. Curr. Opin. Immunol. 55, 31-37 (2018)

60. Yamada, A., Miyakawa, Y. \& Kosaka, K. Entrapment of anti-DNA antibodies in the kidney of patients with systemic lupus erythematosus. Kidney Int. 22, 671-676 (1982).
61. Clynes, R., Dumitru, C. \& Ravetch, J. V. Uncoupling of immune complex formation and kidney damage in autoimmune glomerulonephritis. Science $\mathbf{2 7 9}$ 1052-1054 (1998)

62. Qureshi, F. et al. Anti-DNA antibodies cross-reacting with laminin inhibit trophoblast attachment and migration: implications for recurrent pregnancy loss in SLE patients. Am. J. Reprod. Immunol. 44, 136-142 (2000).

63. Mason, L. J., Ravirajan, C. T., Rahman, A., Putterman, C. \& Isenberg, D. A. Is alphaactinin a target for pathogenic anti-DNA antibodies in lupus nephritis? Arthritis Rheum. 50, 866-870 (2004).

64. Seredkina, N., Van Der Vlag, J., Berden, J., Mortensen, E. \& Rekvig, O. P. Lupus nephritis: enigmas, conflicting models and an emerging concept. Mol. Med. 19, 161-169 (2013).

65. Madaio, M. P. et al. Murine monoclonal anti-DNA antibodies bind directly to glomerular antigens and form immune deposits. J. Immunol. 138, 2883-2889 (1987).

66. Kazama, $\mathrm{H}$. et al. Induction of immunological tolerance by apoptotic cells requires caspase-dependent oxidation of high-mobility group box-1 protein. Immunity 29, 21-32 (2008).

67. McGaha, T. L., Sorrentino, B. \& Ravetch, J. V. Restoration of tolerance in lupus by targeted inhibitory receptor expression. Science 307, 590-593 (2005).

68. Casciola-Rosen, L. A., Anhalt, G. \& Rosen, A. Autoantigens targeted in systemic lupus erythematosus are clustered in two populations of surface structures on apoptotic keratinocytes. J. Exp. Med. 179, 1317-1330 (1994).

69. Casciola-Rosen, L., Rosen, A., Petri, M. \& Schlissel, M. Surface blebs on apoptotic cells are sites of enhanced procoagulant activity: implications for coagulation events and antigenic spread in systemic lupus erythematosus. Proc. Natl Acad. Sci. USA 93, 1624-1629 (1996).

70. Rother, N., Pieterse, E., Lubbers, J., Hilbrands, L. \& van der Vlag, J. Acetylated histones in apoptotic microparticles drive the formation of neutrophil extracellular traps in active lupus nephritis. Front. Immunol. 8, 1136 (2017).

71. Sisirak, V. et al. Digestion of chromatin in apoptotic cell microparticles prevents autoimmunity. Cell 166, 88-101 (2016).

72. Fuchs, T. A., Brill, A. \& Wagner, D. D. Neutrophil extracellular trap (NET) impact on deep vein thrombosis. Arterioscler. Thromb. Vasc. Biol. 32 1777-1783 (2012).

73. Fuchs, T. A. et al. Extracellular DNA traps promote thrombosis. Proc. Natl Acad. Sci. USA 107, 15880-15885 (2010).

74. Warnatsch, A., loannou, M., Wang, Q. \& Papayannopoulos, V. Inflammation. Neutrophil extracellular traps license macrophages for cytokine production in atherosclerosis. Science 349, 316-320 (2015). 